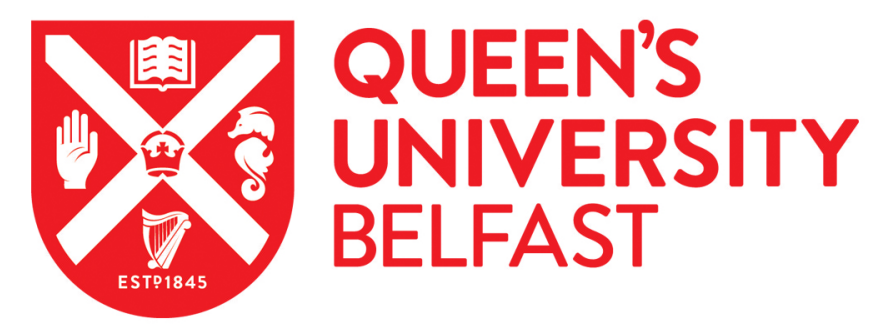

\title{
Laughter Induction Techniques Suitable for Generating Motion Capture Data of Laughter Associated Body Movements
}

McKeown, G., Curran, W., McLoughlin, C., Griffin, H. J., \& Bianchi-Berthouze, N. (2013). Laughter Induction Techniques Suitable for Generating Motion Capture Data of Laughter Associated Body Movements. In 2013 10th IEEE International Conference and Workshops on Automatic Face and Gesture Recognition (FG) (pp. 1-5). Institute of Electrical and Electronics Engineers Inc.. https://doi.org/10.1109/FG.2013.6553806

Published in:

2013 10th IEEE International Conference and Workshops on Automatic Face and Gesture Recognition (FG)

Document Version:

Early version, also known as pre-print

Queen's University Belfast - Research Portal:

Link to publication record in Queen's University Belfast Research Portal

Publisher rights

(c) 2013 IEEE. Personal use of this material is permitted. Permission from IEEE must be obtained for all other uses, in any current or future media, including reprinting/republishing this material for advertising or promotional purposes, creating new collective works, for resale or redistribution to servers or lists, or reuse of any copyrighted component of this work in other works.

\section{General rights}

Copyright for the publications made accessible via the Queen's University Belfast Research Portal is retained by the author(s) and / or other copyright owners and it is a condition of accessing these publications that users recognise and abide by the legal requirements associated with these rights.

Take down policy

The Research Portal is Queen's institutional repository that provides access to Queen's research output. Every effort has been made to ensure that content in the Research Portal does not infringe any person's rights, or applicable UK laws. If you discover content in the Research Portal that you believe breaches copyright or violates any law, please contact openaccess@qub.ac.uk. 


\title{
Laughter Induction Techniques Suitable for Generating Motion Capture Data of Laughter Associated Body Movements
}

\author{
Gary McKeown, William Curran, Ciaran McLoughlin, Harry J. Griffin, Nadia Bianchi-Berthouze
}

\begin{abstract}
Laughter is a frequently occurring social signal and an important part of human non-verbal communication. However it is often overlooked as a serious topic of scientific study. While the lack of research in this area is mostly due to laughter's non-serious nature, it is also a particularly difficult social signal to produce on demand in a convincing manner; thus making it a difficult topic for study in laboratory settings. In this paper we provide some techniques and guidance for inducing both hilarious laughter and conversational laughter. These techniques were devised with the goal of capturing motion information related to laughter while the person laughing was either standing or seated. Comments on the value of each of the techniques and general guidance as to the importance of atmosphere, environment and social setting are provided.
\end{abstract}

\section{INTRODUCTION}

Recently, attention has turned to a much overlooked aspect of human social interaction - laughter. Given the frequency with which laughter occurs in human conversation and human interactions there has been a tendency to overlook its importance as a human social signal. No doubt this has been due to its association with humour and frivolity, making it appear to be an inappropriate focus for the serious nature of scientific enquiry. However this perception has begun to change and laughter is now at the centre of some extensive scientific scrutiny. This is driven by those who view laughter as an important component of well-being and a likely contributor to a healthy lifestyle, those who wish to understand its role in social bonding and social exclusion, and of course those interested in the links between humour and laughter.

A first major distinction between types of laughter seems to be between the types of laughter that are associated with some directly humorous event - sometimes termed hilarious laughter [1], and the types of laughter that serve as social signals within human social interactions - sometimes termed social or conversational laughter [2], [3]. Understanding when these kinds of laughter occur, and being able to recognise them and synthesise them have become important considerations within research in the field of social signals. Before serious study of laughter can take place - including its recognition and synthesis - sufficient amounts of data are required for the training of algorithms; this data should also allow the generation of models that can be tailored to

The research leading to these results has received funding from the European Union Seventh Framework Programme (FP7/2007-2013) under grant agreement no. 270780 (ILHAIRE project).

G. McKeown and W. Curran are with the School of Psychology, Queen's University Belfast, UK. $g$. mckeown@ qub.ac.uk

C. McLoughlin, H. J. Griffin, N. Bianchi-Berthouze are with UCL interaction Centre, UCL, UK. a variety of laughter situations. A number of databases exist which seek to address this issue; including the AVLaughter Cycle database [4], The MAHNOB Laughter database [5] and the ILHAIRE Laughter database [6]. These databases provide a welcome addition to the body of data that focus on laughter; however, they concentrate mainly on seated participants with particular emphasis placed on the face and facial expressions. In addition to involving distinct acoustic phenomena and having a strong impact on facial expressions, laughter is also a social signal with a strong component of body movement. Movements associated with laughter include respiratory movements of the chest; gross body movements such the "doubling up" (where the laugher leans over); gestural hand movements such as slapping or covering the mouth and face; and changes in proximity between interlocutors. These sources of information are best captured with motion capture equipment.

There are a lot of challenges in capturing such rich body movement data in laughter situations. Laughter is not an easy subject of study and, although people laugh a lot in their everyday interactions, it is a very difficult behaviour to reproduce on demand; rather, demand-driven laughter quickly degenerates into laughter that can appear apparently false and unbelievable. These factors make it a particularly difficult behaviour on which to gather data. This paper seeks to provide information on laughter induction and capture techniques that the authors have found useful in our goal of generating and recording sustainable levels of laughter over a prolonged data gathering period. We report on two sessions of data gathering using two different motion capture techniques.

\section{ATMOSPHERE, ENVIRONMENT AND SOCIAL COMPANY}

As genuine laughter is an elusive social signal to generate on demand, but very common in natural settings, the first lesson we learned in our attempts to generate it was the importance of creating a social setting that is conducive to laughter generation. We sought to break the formality of the laboratory environment by putting on background music and by encouraging those in the laboratory to join in and chat and generate a generally jovial atmosphere. In motion capture experiments a substantial amount of time is necessarily spent setting up the equipment. The inherent absurdity of fixing gyroscopes or visual markers to the participants was highlighted and often became a source of amusement itself.

Because a number of studies have shown that laughter is much more common among closely connected social 
groups [7], we recruited pairs of friends for the two laughterinducing sessions.

\section{LAUGhter Induction TEChNiQUeS}

\section{A. Session 1}

The laughter-inducing techniques relied on social interactions in groups, and included: reading taboo tongue twisters, watching funny internet videos, playing competitive partystyle games, and playing social computer games. An important factor in ensuring that laughter would be readily induced was constructing the testing environment. To make laughter more likely we created a relaxed atmosphere and sought to minimize tension in the participants. To achieve these environmental circumstances we had background music playing and had 5 to 10 people in each social grouping, which served to remove direct focus from the volunteer participants. The vocal laughter sounds were captured using head mounted microphones and the volume of the music was low so that it did not interfere with this capture - if such equipment is not available music is probably not advisable. The social group was made up of other researchers from the ILHAIRE project in addition to the dyad under study. All members of a social grouping participated in the various social activities, thus ensuring that volunteer participants did not feel they were the focus of attention.

1) Motion Capture System: A Qualysis motion capture system - an optical motion capture system with retroreflective markers placed on key points on the body - was used to record participants' movements. The motion capture system utilised 18 cameras over a large volume normally used for gathering sports related motion capture data. Two marker placement schemes were used, one more extensive than the other. The placement of the two marker schemes are depicted in Figure 1. In addition to the motion capture system, two high quality video cameras were used to record the sessions.

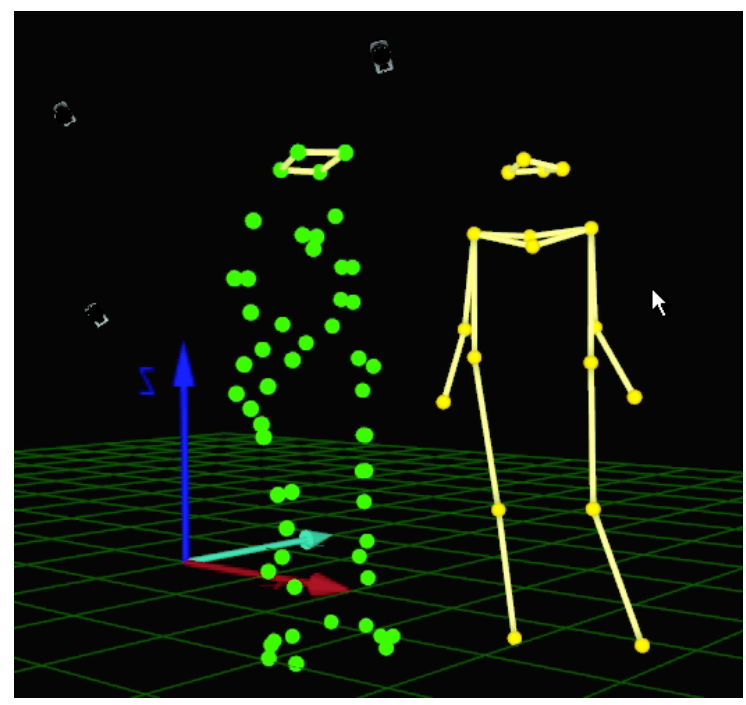

Fig. 1. Motion Capture single frame of a standing interacting dyad captured using the Qualysis motion capture system
TABLE I

MEETING 1: LAUGHTER ELICITATION MATERIALS USED DURING DATA GATHERING TRIALS AT SESSION 1.

\begin{tabular}{|c||l||c|}
\hline \hline Posture & Task & $\begin{array}{c}\text { Target } \\
\text { laughter elicitation }\end{array}$ \\
\hline Standing & Charades/EmoTABOO & Hilarious \\
& Limericks & Hilarious \\
& Games (Bopit) & Hilarious \\
& Dancing Games (Wii) & Hilarious \\
& Role Play (Catwalk) & Hilarious \\
& Tongue twisters & Hilarious \\
& Box Tasks & Social \\
\hline Sitting & YouTube Videos & Social, hilarious \\
& YouTube Journey & Social, hilarious \\
\hline \hline
\end{tabular}

2) Participants: Eight participants in four pairs took part in the sessions. The pairs were same sex dyads and all were friends with the other member of the pair. Sessions were approximately 3 hours. These began with calibration of the system and time to fix the adhesive reflective markers.

3) Tasks: Video and motion capture recording was continuous. The advantage of continuous recording was that it gave the opportunity to capture laughing behaviour during the rest periods between laughter-inducing tasks as well as during the tasks themselves. While the tasks typically induced hilarious laughter, the interactions between tasks resulted in conversational laughter being produced. The tasks were in general party game style tasks, the kind of games that would be played at informal social gatherings and family occasions. In these tasks the participants could be either the focus of the task or a spectator. Participants were instructed to stay within the motion capture volume during rest periods, thus ensuring that episodes of conversational laughter would be captured.

Eight participants were recruited for the session, and were paid for their participation. Participants carried out tasks that required either standing or sitting. In the 'standing' tasks participants were encouraged to move about the motion capture volume freely unless constrained by the requirements of a task. A list of the tasks used in Session 1 can be seen in Table I. The first of these "standing tasks" was a Charades style task where a mime had to act and describe a word without using a list of taboo words. This was based on a task used in the EmoTaboo protocol [8]. This task did not generate laughter and, as we used it early in the process, we dropped this task from the repertoire early on.

A task that successfully induced laughter and served well as a first task and "ice-breaker" for the session was the tongue twister task; which involves reading aloud phrases, sentences or rhymes that contain difficult to pronounce word sequences often involving alliteration and quick repetition of words. A certain class of tongue twister encourages or tricks the participant into uttering swear words against their intention; these "rude" tongue twisters were more effective laughter elicitors than tongue twisters without the swearing component. A list of effective tongue twisters can be found in Table II. 
TABLE II

TONGUE TWISTERS USED IN ONE OF THE LAUGHTER-INDUCING TASKS

\begin{tabular}{|c|}
\hline I am not the pheasant plucker, \\
I'm the pheasant plucker's mate. \\
I am only plucking pheasants \\
Because the pheasant plucker's late. \\
One smart fellow; he felt smart. \\
Two smart fellows; they felt smart. \\
Three smart fellows; they all felt smart. \\
\hline I slit the sheet, the sheet I slit; \\
and on the slitted sheet I sit. \\
\hline A flea and a fly flew up in a flue. \\
Said the flea, "Let us fly!" \\
Said the fly, "Let us flee!" \\
So they flew through a flaw in the flue. \\
\hline Silly Simon's sitting in a shoe shine shop. \\
Where he sits he shines, \\
and where he shines he sits. \\
\hline Sally on the seashore saw a ship in sight \\
\hline
\end{tabular}

In a similar vein we had a task that involved reading Limericks that were taboo in nature. These did not, however, result in the same level of success as the tongue twisters; so it did not appear to be simply the taboo nature of the material that created laughter but some interaction between the social grouping, the atmosphere and the task that resulted in laughter.

Another kind of task we used was competitive games. These involved participants either playing against each other or spectating and watching others compete. In one of these tasks we had participants play the commercial game "Bop it"- a game which requires rapid responses from the players. This worked moderately well in generating laughter but better in two of the pairs than in the other two.

Another competitive activity used a computer game Nintendo Wii Dance III - in which players took turns in dancing to music and following the on-screen dancing instructions, while others spectated. This proved to be very successful in generating laughter.

Another task was to encourage a role play scenario. We set up a scenario in which the participants and experimenters walked along an imaginary catwalk. This act, the level of ridiculousness and the concurrent loss of dignity it produced resulted in the generation of strong bouts of laughter.

We also sought to induce laughter while participants were seated. To avoid over constraining their movements participants were provided with a comfortable low level stool with no back, thus allowing them to move backwards to the extent they desired while laughing. In these tasks we used two variants of an online video watching task. In one of these we showed the participants pre-prepared humorous clips from YouTube. This was moderately successful at inducing laughter. In a second variant of this task, which we termed "YouTube journey", the participants guided the selection of YouTube clips. This proved to be very effective in generating laughter.

4) Conversational Laughter: Although the active laughter induction techniques in the tasks focused on gathering data related to hilarious laughter, we also sought to gather conver- sational or social laughter. Numerous instances of this kind of laughter was produced in the breaks between tasks, when participants engaged in natural conversational behaviour and associated laughter. Because we had opted to use continuous motion capture and video recording, we were able to capture these laughter events and generated a rich database of conversational laughter. An interesting observation was that participants would often complain that they could not laugh any more during the tasks and complained of fatigue in relation to laughter, yet immediately returned to generating laughter during the inter-tasks breaks.

5) Motion Capture data: Session 1 generated 101 Qualisys Track Manager files ranging in size from 26.5 MB to $405 \mathrm{MB}$, giving a total of $27.87 \mathrm{~GB}$ of motion capture data. The motion capture data alongside audio data and video data has been made available as part of the ILHAIRE database [6] for broad usage by the research community.

6) Other Tasks: We used a variety of other tasks in this session that did not work well. We had hoped to induce social laughter by having participants negotiate who places their hand inside a box-based on tasks from [9]-but this did not generate laughter; the EmoTaboo and Limericks tasks were also of limited use and were quickly discarded. In general the tasks either worked reasonably well or did not work at all; whether a task will work or not is very hard to predict, but when it does not generate laughter this becomes evident at an early stage. In general the advice here is to have many tasks prepared and abandon them readily if they appear not to be working. To over laboriously pursue a given task is likely to result in a deadening of the atmosphere.

\section{B. Session 2}

A second session of data capture took place in a different laboratory building, and was guided by the lessons learnt from the first session.

1) Motion Capture System: This session used the Animazoo IGS190 motion capture system-an inertial motion capture system. The default configuration of this system involves wearing a series of small, lightweight gyroscopes that are attached over the participant's clothing using Velcro straps. There are 17 gyroscopes used, with each fitting to a different part of the participant's body, computing the angles and offsets in 3D space between the gyroscopes for each of their limbs, head, and torso, and so on in relation to each other. In this session we wished to capture fine shoulder movements as this was missing in the first session and was deemed important information with respect to laughter [10]. The default configuration of the motion capture suit was adjusted to account for this requirement. Data gathering took place in large, quiet classrooms with flexible furniture that was moved to the sides of the room, affording participants plenty of space to move about. The equipment layout of the room was slightly different for the standing and seated tasks.

2) Participants: Eighteen participants were recruited in pairs, with movement data collected from one member of each pair as only one participant per pair wore the motion capture system. The motion capture wearing participants 
TABLE III

MEETING 2: LAUGHTER ELICITATION MATERIALS USED DURING DATA GATHERING TRIALS SESSION 2.

\begin{tabular}{|c||l||c|}
\hline \hline Trial Section & Task Sequence & Target laughter \\
\hline Standing & Tongue twisters. & Hilarious, awkward \\
& mini-break & Social \\
& YouTube video & Hilarious, social \\
& mini-break & Social \\
& YouTube video & Hilarious \\
& mini-break & Social \\
& Puerile song titles & Hilarious, social \\
\hline Break & Chat with researchers & Social. \\
\hline Sitting & Pictionary. & Social, hilarious \\
& mini-break & Social \\
& Videos shown to one participant & Hilarious, awkward \\
& while second watches & Social \\
& mini-break & Awkward, hilarious \\
& YouTube video & Social \\
\hline Thini-break & Perform fake laughter on request & Fake \\
\hline \hline
\end{tabular}

comprised three males and six females; their mean age was 25.7. Participants were again paid for their participation. Participants were drawn from a mix of cultures, including Western European, East Asian, North American and South Asian.

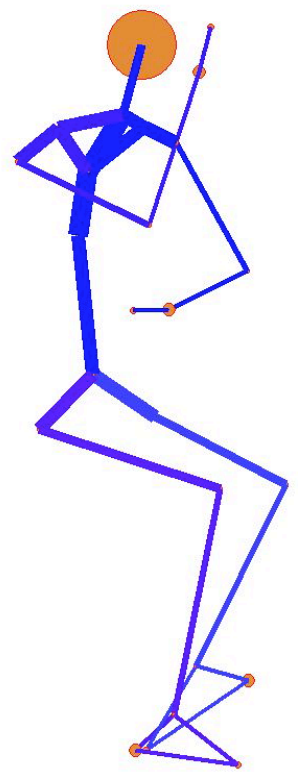

Fig. 2. Motion Capture single frame of a seated laughing participant captured using a Animazoo motion capture systems

3) Tasks: Table III shows the tasks and the sequence used in Session 2. The "Bop it" task was used in a pilot phase but proved unsuccessful in inducing laughter and was therefore abandoned at that stage. This contrasts with the successful deployment of this task in Session 1, and again highlights that different factors other than the task or stimuli used can influence the likelihood of successful laughter induction.

The first task once again consisted of participants reading tongue twisters; this time they read them directly to one another taking alternating turns, with the instruction to try to get through as many as possible in five minutes. Two versions of the tongue twisters were used: one used for six of the sessions in English and, for those sessions with East Asian participants, a separate list of Mandarin Chinese language tongue twisters was used. It was felt that this would be more successful than using English tongue twisters for this population. Participants were advised not to worry about getting the tongue twisters correct, but just to move through them as fast as possible.

The second task was a YouTube task that differed in the fact that participants were standing side by side rather than being seated whilst watching pre-prepared videos on YouTube. The videos shown had been pre-selected as likely to induce laughter. This was sometimes due to the amusing nature of the video, for example a man with a highly "contagious" laugh. Other videos used in-jokes or internet "memes" and so we would caution that these are highly culturally and demographic dependent.

A further task was developed in this session, again around the theme of introducing swear words into non-offensive language. In this task participants read a list of song titles, all including the word "love". Participants were asked to replace the word "love" with a swear word. This resulted in puerile phrases but was successful in eliciting hilarious laughter.

The next set of tasks involved the participants sitting down facing each other, with a computer monitor in front of each. Both monitors were connected to one desktop and the display was mirrored on each screen so that both participants could see the same picture on both displays.

The first of the seated tasks was based on the commercial game Pictionary. Participants had to pick a clue from many, out of a cup, written on a small piece of paper. They were not allowed to disclose what was on the paper to the second participant, but rather had to draw clues for their partner using Microsoft Paint, which the other participant could see on their monitor. The task and environment were designed with the goal of eliciting both hilarious and social laughs. The participant pairs produced prolonged bouts of laughter (approx. 15 to 20 seconds) throughout this exercise. Indeed much of the communication between participants as they guessed what the other was drawing took place predominantly with laughter and some gestures, but not very many words. An example of this is when the drawing participant would answer a guess from their partner with a laugh, the tone of which was meant to convey whether the guess was correct or not.

An alternate version of the YouTube task was created for this session. In this task participants were alternately shown amusing videos (often of animals behaving in amusing ways) one at a time, with the other participant asked to look at the face of the participant viewing the amusing stimulus. The second participant could not see the stimulus, but as the viewing of the stimulus was passed back and forth, it was 
hoped that this would stimulate more hilarious laughter. This was only occasionally successful and was soon abandoned as a task.

In a final task, participants were asked to view a video on YouTube. This video depicted sequences of home video style footage from all over the world, generally showing people suffering pratfalls or some personal embarrassment or injury. Generally speaking, the tone of the video is designed to cause amusement and hilarity but also some level of discomfort, as the viewer is aware that many of the people at which they are laughing could probably have suffered painful injuries as a result of these falls and mishaps. However, the video is cut to show a baby laughing in between each segment. This results in many viewers laughing, but often some sense of restraint, as if the participants experienced a pang of guilt when viewing another's misfortune and laughing inappropriately.

4) Conversational Laughter: Once again in this session participants were given short breaks between each of the tasks. Participants were allowed to discuss what they had just completed and were engaged in general conversation, under the pretence of needing time to set up for the next task. However, recording continued, and at this point several conversational and back-channelling laughs were observed whilst the participants engaged in conversation with the researchers and between themselves. These laughs were generally classified as social or conversational laughs.

5) Fake Laughter: Throughout both standing and sitting trials, participants were periodically requested to produce a fake laugh. Participants were asked to imagine what they thought a grand, overblown, "theatrical" laugh might be, and to produce that. Further, they were also asked to imagine a social situation with an imbalanced power element, such as with one of their university lecturers or their boss, where the more powerful person had said something that they themselves thought was funny, but that the participant did not. Participants were asked to imagine they had to laugh at what had been said out of politeness and to show respect and to ensure the listener cannot detect the laugh is disingenuous. They were then asked to produce the laugh.

6) Motion Capture data: In total 126 data files were generated containing movies of the stick figure nature shown in Figure 2. These files range in size from 1.7 to $20 \mathrm{MB}$ and and the collective size of the files is 946.2 MB.This data has been made available as part of the ILHAIRE database [6] for broad usage by the research community.

\section{General Discussion}

We have described a range of new laughter-inducing techniques that have proven to be effective tools with which to induce laughter. Using these new laughter-induction techniques we have accumulated approximately 240 minutes of laughter-rich material.

The main lesson learnt from this exercise was that it is not a straight forward matter to generate sustained levels of spontaneous and natural laughter. We have found that a number of environmental and social factors play an important role in creating a setting conducive to laughter generation.
A friendly, relaxing environment is a particularly important consideration for putting participants at ease in what is, essentially, a research environment. We achieved this by playing popular music in the background and ensuring that there were additional people, other than the participants, in the motion capture space. A second important factor is to ensure that participants do not feel they are the focus of attention; this was achieved by having members of the team also participate in the various tasks. Thus participants were just as often spectators as being players. It is also important that the session is not over-choreographed; that is, an element of spontaneity and willingness to change tack at short notice is an effective approach to avoiding a subdued mood setting in. A variety of tasks with frequent breaks is also an effective means of sustaining laughter generation. Indeed, if one uses the approach of continuous recording, the inter-tasks breaks can be a rich source of laughter material. While all of these factors are important, arguably the most important factor influencing the production of laughter is the relationship that the participants share. In other words, if one wishes to generate laughter successfully, this is most likely to be achieved if the participants already have a strong social relationship (i.e. they are friends).

\section{REFERENCES}

[1] R. Niewiadomski, J. Urbain, C. Pelachaud, and T. Dutoit, "Finding out the audio and visual features that influence the perception of laughter intensity and differ in inhalation and exhalation phases," in Proceedings of 4th International Workshop on Corpora for Research on Emotion, Sentiment \& Social Signals, LREC, 2012.

[2] E. Holt, "The last laugh: Shared laughter and topic termination," Journal of Pragmatics, vol. 42, no. 6, pp. 1513-1525, 2010.

[3] — - "On the nature of 'laughables': laughter as a response to overdone figurative phrases," Pragmatics, vol. 21, no. 3, pp. 393-410, 2011.

[4] J. Urbain, E. Bevacqua, T. Dutoit, A. Moinet, R. Niewiadomski, C. Pelachaud, B. Picart, J. Tilmanne, and J. Wagner, "The avlaughtercycle database," in Proceedings of the Seventh International Conference on Language Resources and Evaluation (LREC'10), N. C. C. Chair), K. Choukri, B. Maegaard, J. Mariani, J. Odijk, S. Piperidis, M. Rosner, and D. Tapias, Eds., 2010.

[5] S. Petridis, B. Martinez, and M. Pantic, "The MAHNOB Laughter Database," Image and Vision Computing, 2012.

[6] G. McKeown, R. Cowie, W. Curran, W. Ruch, and E. Douglas-Cowie, "ILHAIRE Laughter Database," in Eighth international conference on Language Resources and Evaluation (LREC 2012), 2012, pp. 1-4.

[7] M. Mehu and R. I. M. Dunbar, "Naturalistic observations of smiling and laughter in human group interactions," Behaviour, vol. 145, no. 12, pp. 1747-1780, 2008.

[8] A. Zara, V. Maffiolo, J. Martin, and L. Devillers, "Collection and annotation of a corpus of human-human multimodal interactions: Emotion and others anthropomorphic characteristics," Affective Computing and Intelligent Interaction, pp. 464-475, 2007.

[9] I. Sneddon, M. McRorie, G. McKeown, and J. Hanratty, "The Belfast Induced Natural Emotion Database," IEEE Transactions on Affective Computing, vol. 3, no. 1, pp. 32-41, 2012.

[10] M. Mancini, G. Varni, D. Glowinski, and G. Volpe, "Computing and Evaluating the Body Laughter Index," in Third International Workshop on Human Behavior Understanding, IEEE/RSJ International Conference on Intelligent Robots and Systems, 2012, pp. 1-9. 